\title{
Efectos de diferentes relaciones energía/proteína sobre el comportamiento productivo de pollos parrilleros machos*
}

\author{
Sindik, M..'; Revidatti, F.'; Terraes, J.'; Fernández, R. ${ }^{1}$; Sandoval, G. ${ }^{2}$ \\ ${ }^{1}$ Cátedras Producción de Aves y ${ }^{2}$ Bioquímica, Facultad de Ciencias Veterinarias, UNNE, Sargento \\ Cabral 2139, Corrientes (3400), Tel/Fax: 54-3783-425753, E-mail: granja@vet.unne.edu.ar.
}

\begin{abstract}
Resumen
Sindik, M.; Revidatti, F.; Terraes, J.; Fernández, R.; Sandoval, G.: Efectos de diferentes relaciones energía/proteína sobre el comportamiento productivo de pollos parrilleros machos. Rev. vet. 20: 2, 121-125, 2009. Se evaluaron en este estudio los efectos de dos dietas isocalóricas con diferentes niveles de proteína sobre el peso corporal, consumo de alimento y conversión alimenticia en pollos parrilleros machos. El tratamiento 1 (T1) proporcionó una relación energía/proteína amplia (dieta tipo forrajera) y el 2 (T2) una relación estrecha (dieta tipo parrillero). Las raciones fueron suministradas ad libitum durante todo el ciclo productivo (49 días), registrándose las variables en forma semanal. No se hallaron diferencias estadísticas significativas para peso corporal en ningún momento del ciclo $(\mathrm{p}=0,92)$, aunque desde el día 21 hasta el final, la media de T1 tendió a presentar valores algo superiores. Los consumos de T1 y T2 arrojaron respectivamente los valores de $5.672 \pm 451 \mathrm{~g}$ y $5.807 \pm 469 \mathrm{~g}(\mathrm{p}=0,76)$. La conversión registró un valor acumulado al final del ciclo de $1,92 \pm 0,02$ y 2,0 $\pm 0,12$ para los tratamientos $\mathrm{T} 1$ y T2, valores que no alcanzaron a ser estadísticamente significativos $(\mathrm{p}=0,31)$. Los resultados obtenidos indican que a pesar de las diferencias en los niveles de proteínas y de las relaciones energía y proteína existentes, el nivel de eficiencia global no fue afectado significativamente durante el período de análisis.
\end{abstract}

Palabras clave: pollo parrillero, alimentación, dietas, eficiencia.

\begin{abstract}
Sindik, M.; Revidatti, F.; Terraes, J.; Fernández, R.; Sandoval, G.: Effects of different energy/protein ratios on the productive performance of male broiler chickens. Rev. vet. 20: $2,121-125,2009$. The effects of two isocaloric diets with different protein levels on body weight, feed intake and feed conversion in male broilers were evaluated. The treatments consisted of the provision of two isocaloric diets with varying protein levels. Treatment 1 (T1) provided a broad energy/protein relation forage-like diet and treatment 2 (T2) a close relation broiler commercial diet. The rations were fed ad libitum during the entire production cycle (49 days) and the variables were recorded weekly. We found no statistically significant differences between treatments for body weight $(\mathrm{p}=0.92)$ at any time of the cycle, but from day 21 until the end of the cycle, the mean of T1 tended to show values slightly higher. Feed consumption showed values of $5,672 \pm 451 \mathrm{~g}$ and $5,807 \pm 469 \mathrm{~g}(\mathrm{p}=0.76)$. Feed conversion ratio recorded a cumulative value at the end of the cycle of $1.92 \pm 0.02 \mathrm{~g}$ and $2.0 \pm 0.12$ for T1 and T2 respectively, being values no statistically significant different $(\mathrm{p}=0.31)$. The results of this trial indicate that, despite the differences in the levels of protein and energy and protein relation, the overall efficiency level was not significantly affected for the considered period of time.
\end{abstract}

Key words : broiler chicken, food, diets, efficiency.

\section{INTRODUCCIÓN}

En las producciones que utilizan especies de rápido crecimiento, entre las cuales las aves de corral ocupan un lugar destacado, el rubro alimentación representa dentro de los costos operativos aproximadamente el $70 \%$ del total, por lo cual su ajuste significa muchas

*SGCyT-UNNE PI 108/2005

Recibido: 7 agosto 2009 / Aceptado: 4 setiembre 2009 veces la diferencia entre el éxito y el fracaso de un emprendimiento ${ }^{12}$.

Por lo general, para garantizar el logro de los objetivos de la producción de carne (velocidad de crecimiento, conversión alimenticia, conformación, rendimiento de la canal), es necesario el uso de raciones balanceadas de alta densidad nutricional, las cuales convenientemente suministradas a lo largo del ciclo de producción, posibilitan que los pollos expresen su potencial genético ${ }^{16}$. 
El mejoramiento genético y los programas de alimentación del pollo parrillero llevaron a un incremento progresivo en su eficiencia productiva ${ }^{2}$. Aceptando que existen diferencias mínimas en las tasas de conversión entre distintas estirpes (factor genético), la mejora en dicha variable estaría asociada a la disminución en los requerimientos de mantenimiento (como consecuencia de un ciclo de menor duración), o al empleo de raciones balanceadas de baja densidad nutricional que en algunos casos se utilizan para mejorar la viabilidad del lote. En función de los objetivos productivos y las condiciones de explotación en general, dichas dietas pueden alcanzar niveles de eficiencia técnica y económica que justifican su empleo.

En tanto se logre la misma calidad y cantidad de producto, con menor consumo de alimento y/o con la ingestión de una dieta más económica, las condiciones de rentabilidad de la operación habrán mejorado ${ }^{5,6}$. La curva de crecimiento de los parrilleros alimentados ad libitum tiene una forma sigmoidea ${ }^{18}$, mientras que las aves bajo restricción alimenticia manifiestan una disminución en su tasa de crecimiento al inicio, lo cual reduce los requerimientos de mantenimiento totales del ave y por tanto se registra una mejora de la conversión alimenticia si se alcanza el mismo peso en igual tiempo ${ }^{8}$.

El objetivo del presente ensayo fue evaluar el peso corporal, consumo de alimento y conversión alimenticia en un ciclo de 49 días en pollos para carne (machos) pertenecientes a una línea genética comercial autosexante (gen de emplume lento) en los cuales se trabajó con dos tipos de dietas, una de ellas con una densidad nutricional baja, lo que representó un esquema de restricción cualitativa respecto a la otra dieta cuya composición se asimilaba a las dietas comerciales convencionales para pollos parrilleros.
Tabla 1. Composición química de la ración de iniciación.

\begin{tabular}{lccccc}
\hline componente & unidad & $\mathrm{T} 1$ & base seca & $\mathrm{T} 2$ & base seca \\
\hline energía met. & $\mathrm{kcal} / \mathrm{kg}$ & 3000 & 3656 & 3000 & 3656 \\
calcio & $\%$ & 0,95 & 1,69 & 0,95 & 1,66 \\
fósforo disp. & $\%$ & 0,48 & 0,81 & 0,48 & 0,95 \\
proteína & $\%$ & 20,0 & 20,73 & 22,7 & 22,23 \\
grasa & $\%$ & 4,77 & 7,65 & 5,43 & 8,62 \\
fibra & $\%$ & 3,40 & 3,45 & 3,66 & 3,82 \\
ac linoleico & $\%$ & 2,28 & & 2,43 & \\
lisina & $\%$ & 1,17 & & 1,22 & \\
metionina & $\%$ & 0,52 & & 0,49 & \\
met + cistina & $\%$ & 0,84 & & 0,85 & \\
triptófano & $\%$ & 0,26 & & 0,26 & \\
arginina & $\%$ & 1,33 & & 1,54 & \\
isoleucina & $\%$ & 1,03 & & 1,13 & \\
treonina & $\%$ & 0,79 & & 0,89 & \\
xantofila & $\mathrm{mg} / \mathrm{kg}$ & 19,00 & & 17,00 & \\
colina & $\mathrm{mg} / \mathrm{kg}$ & 1212,6 & & 1349 & \\
relación E:P & $\mathrm{ener} / \mathrm{prot}$ & 150 & 176,36 & 132,15 & 164,46 \\
\hline
\end{tabular}

Tabla 2. Composición química de la ración de terminación.

\begin{tabular}{lccccc}
\hline componente & unidad & $\mathrm{T} 1$ & base seca & $\mathrm{T} 2$ & base seca \\
\hline energía met. & $\mathrm{kcal} / \mathrm{kg}$ & 3150 & 3752 & 3150 & 3807 \\
calcio & $\%$ & 0,95 & 1,52 & 0,95 & 1,33 \\
fósforo disp. & $\%$ & 0,47 & 0,79 & 0,47 & 0,75 \\
proteína & $\%$ & 18,5 & 18,29 & 20,0 & 19,52 \\
grasa & $\%$ & 6,44 & 10,36 & 7,0 & 10,34 \\
fibra & $\%$ & 3,1 & 4,03 & 3,3 & 4,15 \\
ac linoleico & $\%$ & 3,18 & & 3,5 & \\
lisina & $\%$ & 0,98 & & 1,1 & \\
metionina & $\%$ & 0,45 & & 0,45 & \\
met + cistina & $\%$ & 0,76 & & 0,78 & \\
triptófano & $\%$ & 0,25 & & 0,27 & \\
arginina & $\%$ & 1,23 & & 1,35 & \\
isoleucina & $\%$ & 0,93 & & 1,00 & \\
treonina & $\%$ & 0,75 & & 0,80 & \\
xantofila & $\mathrm{mg} / \mathrm{kg}$ & 25 & & 24,16 & \\
colina & $\mathrm{mg} / \mathrm{kg}$ & 1128 & & 1219 & \\
relación E:P & ener/prot & 170,2 & 205,14 & 150 & 195,03 \\
\hline
\end{tabular}

\section{MATERIAL Y MÉTODOS}

Los ensayos se llevaron a cabo en un galpón cerrado con techo de cinc, paredes de mampostería, ventanas laterales, cielorraso de material aislante y piso de cemento alisado. El ciclo de producción tuvo una duración de 49 días y fue dividido en dos etapas: inicio ( 0 a 21 días) y terminación (21 días a faena).

El agua fue provista por un bebedero de plato con recipiente invertido con capacidad de 4 litros $(60 \mathrm{~cm}$ de perímetro) a razón de uno por compartimiento $(4,3 \mathrm{~cm}$ lineales por ave). Se utilizó un comedero tolva de 6 kilos de capacidad ( $125 \mathrm{~cm}$ de perímetro) por cada corral $(8,9 \mathrm{~cm}$ lineales por ave). El piso fue cubierto con cama de cáscara de arroz de $5 \mathrm{~cm}$ de espesor, que se repuso parcialmente según necesidad a lo largo del ciclo. Como fuente de calor para los primeros 20 días de vida se em- plearon estufas eléctricas, verificando la temperatura y humedad del ambiente mediante un higrotermómetro digital (Hygrotherm ${ }^{\circledR}$ TFA, Alemania).

Se trabajó con un fotoperíodo natural, manteniendo por la noche y durante la cría una luz piloto de baja intensidad localizada fuera del área de crianza. Se emplearon 196 pollos parrilleros machos, divididos al azar en dos grupos experimentales que fueron alojados en 14 compartimientos o corrales de $2 \mathrm{~m}^{2}$ cada uno, siete corrales por cada tratamiento (densidad $=7$ aves por $\mathrm{m}^{2}$ ). Semanalmente, durante todo el ciclo de producción, se obtuvieron las siguientes variables:

-peso corporal: en cada unidad experimental se obtuvo un promedio de peso vivo correspondiente al corral a partir de los registros de pesos individuales (en g) de cada una de las aves; 
- consumo de alimento: total de alimento consumido (en g) en cada corral, dividido por el número de aves; este cálculo se llevó a cabo mediante el pesaje del remanente que permanecía en los comederos en el día del muestreo, obteniendo el consumo de la semana restando la cantidad ofrecida al inicio de la misma;

-conversión alimenticia: se obtuvo dividiendo el consumo de alimento por el peso corporal.

El diseño experimental permitió evaluar el efecto de las variaciones cualitativas de la dieta: un alimento con una mayor relación energía/proteína (T1) y otro cuya relación era más estrecha dado su mayor proporción de proteína bruta (T2). Las relaciones energía/ proteína del alimento iniciador fueron 150 (T1) / 132 (T2) y para el terminador 170 (T1) / 150 (T2). Tanto la dieta de inicio como la de terminación fueron isocalóricas para cada tratamiento. La composición de estas raciones se presenta en las Tablas 1 y 2 .

Se utilizó un modelo experimental completamente aleatorizado (DCA), en el que cada corral constituyó una unidad experimental y a su vez, una repetición simple de cada tratamiento. El número de repeticiones fue estimado en base al grado de precisión deseado y al desvío estándar de cada variable ${ }^{13}$, en función del comportamiento de las mismas en ensayos previos llevado a cabo por los autores ${ }^{15,17}$. Los valores de las variables se ingresaron en forma categórica en planillas y archivos informáticos para el posterior análisis estadístico utilizando el programa Statistica para Windows. La distribución de todas las variables fue constatada mediante el método de Wilk-Shapiro (Rankit Plot). Se aplicó análisis de la variancia (ANOVA) para DCA y medidas repetidas, evaluando las diferencias entre tratamientos de las variables dependientes considerando límite un nivel de significancia del 5\% 13,17 .

\section{RESULTADOS}

Tal como se observa en Tabla 3, no se registraron diferencias significativas en el consumo de alimento entre los tratamientos a lo largo del ciclo $(p=0,76)$, obteniéndose un consumo promedio acumulado al finalizar el ensayo de $5.672 \pm 451 \mathrm{~g}$ para $\mathrm{T} 1$ y $5.807 \pm 469 \mathrm{~g}$ para T2. Sin embargo y tal como ocurriera a lo largo del ciclo, estas diferencias no alcanzaron a ser significativas.

La evolución de los pesos según tratamiento se presenta en Tabla 4. Se puede observar que la respuesta de ambos grupos experimentales fue similar a lo largo del ciclo, sin acusar diferencias significativas.
En Tabla 5 se observa que no fueron halladas diferencias significativas entre las raciones utilizadas, aunque hacia el final del ciclo (día 42 y 49) se presentaron diferencias numéricas que favorecieron a la ración forrajera.

\section{DISCUSIÓN}

Las dietas de menor densidad nutricional, similares al alimento tipo forrajero del presente ensayo, fueron estudiadas por otros investigadores en etapas tempranas de la vida como estrategia de control del crecimiento de las aves a fin de mejorar la viabilidad al final del ciclo ${ }^{9}$. Algunos autores lograron un patrón de crecimiento más consistente con este método de alimentación, aunque existe controversia respecto a la tasa de consumo, principalmente en función de las características de la dieta empleada y del método de restricción utilizado.

Una investigación demostró crecimiento compensatorio en parrilleros machos de 42 días de edad a los que se le suministró entre los 4 a 11 días una ración con $55 \%$ de ingredientes no digestibles (cáscara de arroz) ${ }^{7}$. Se constató que durante el periodo de suministro del material no digerible, hubo un aumento significativo en el consumo atribuyendo el mismo a las necesidades de las aves de mantener su ingesta energética.

Sin embargo, en otro trabajo no se encontraron diferencias significativas en el consumo con la dilución de la dieta durante la fase de terminación ${ }^{6}$; tales resultados se atribuyeron a la posibilidad que las aves hayan obtenido energía de elementos supuestamente no diges- 
tibles presentes en la dieta. Estos hallazgos coinciden con los resultados obtenidos en el presente estudio, aunque en este caso el suministro de la dieta de menor densidad no se restringió a la fase de terminación.

En otro ensayo se comprobó que el consumo de alimento fue significativamente más alto en dietas que contenían bajos niveles de energía metabolizable 3 , hallándose diferencias cuando los valores energéticos se modificaban, en particular con el suministro de alimento iniciador. Sin embargo, con dietas isocalóricas no hallaron diferencias en el consumo de alimento, incluso variando los niveles de la proteína bruta $(20 \mathrm{y}$ $22 \%$ ) lo cual coincide con los hallazgos realizados por nosotros en el presente ensayo. Los autores del trabajo citado concluyen que la variación en los niveles de proteína bruta presentó efectos no significativos en el consumo de la ración.

Los resultados obtenidos en este ensayo para peso corporal concuerdan con los hallados en otra investigación donde no se observaron diferencias significativas a la faena (42 días) en pollos parrilleros que consumieron una dieta con bajo contenido de proteína bruta ${ }^{4}$. A diferencia de lo observado en nuestro estudio, otros autores destacan que en los experimentos en los cuales los pollos consumieron dietas bajas en proteína bruta, hubo una disminución de la tasa de crecimiento y menor peso corporal al final del período de estudio, por lo que sostienen que dietas bajas en proteína bruta (o altas en la relación energía: proteína) no logran la misma tasa de crecimiento que aquellas con alto nivel proteico y relaciones mas estrechas ${ }^{1}$.

Resultados similares para conversión alimenticia fueron obtenidos en otros trabajos sobre programas de restricción cualitativa en pollos parrilleros. Comparando el rendimiento productivo de una dieta de alta densidad (24\% de proteína bruta y $3.200 \mathrm{kcal} / \mathrm{kg}$ ) y una de baja densidad (19\% de proteína bruta y $2.900 \mathrm{kcal} / \mathrm{kg})$, se comprobó que si bien las aves que consumieron la segunda dieta fueron más livianas a los 42 y 49 días de vida por el menor consumo de alimento, su conversión alimenticia fue similar ${ }^{10}$. Otros diluyeron con un $50 \%$ de cáscara de avena una dieta iniciadora y alimentaron pollos parrilleros de una semana de edad durante 6 días en un programa continuo y otro discontinuo; en ambos se logró el crecimiento compensatorio hacia los 35 días sin diferencia en la conversión alimenticia ${ }^{19}$.

Los resultados obtenidos en el presente ensayo coinciden en general con los autores mencionados, ya que se alcanzaron valores de conversión alimenticia similares para dietas de distintas densidades nutricionales. Independientemente de la trascendencia que posee la cantidad y calidad de la proteína de la dieta respecto a los resultados técnicos productivos, se considera el principio nutricional de mayor costo relativo de las raciones de parrilleros, adjudicándole entre el 40 al 45\% del costo total del alimento formulado ${ }^{14}$. Por esto, la reducción proteica ha sido vista como una de las vías posibles para mejorar los costos de producción y el nivel proteico de la ración pasó a ser definido como el nivel óptimo para responder a las necesidades del ave en aminoácidos ${ }^{11}$.

En conclusión, la falta de diferencias significativas en el consumo, permite aseverar que su modulación no depende solo de la cantidad sino también de la calidad de la proteína bruta suministrada, por lo que se puede afirmar que la dieta de menor concentración en proteína bruta tuvo un adecuado equilibrio en aminoácidos o su digestibilidad fue óptima. La idéntica respuesta en lo que hace a la evolución del peso corporal sugiere que dietas con distintos niveles de proteína bruta tienen una evolución similar cuando el ciclo de producción es largo (49 días). Como el nivel de eficiencia en términos de conversión no fue afectado significativamente durante el período de análisis entre tratamientos, la ración con menor concentración proteica constituye una alternativa a tener en cuenta dado el potencial impacto económico que este hecho puede generar.

\section{REFERENCIAS}

1. Bregendahl K, Sell JL, Zimmerman DR. 2002. Effect of low protein diets on growth performance and body composition of broiler chicks. Poultry Sci 81: 1156-1167.

2. Buxade Carbó C. 1988. El pollo de carne, Ed. MundiPrensa, $2^{\circ}$ ed., Madrid, 365 p.

3. Haq NT, Yaqoob M. 2006. Effect of varying levels of energy and protein on live performance and carcass characteristics. Poultry Sci 43: 388-393.

4. Holsheimer JP, Veerkamp CH. 1992. Effect of dietary energy, protein, and lysine content on performance and yields of two strains of male broiler chicks. Poultry Sci 71: 872-879.

5. Leeson S, Summers JD, Caston LJ. 1991. Diet dilution and compensatory growth in broilers. Poultry Sci 70: 867873.

6. Leeson S, Summers JD, Caston LJ. 1992. Response of broilers to feed restriction or diet dilution in the finisher period. Poultry Sci 71: 2056-2064.

7. Leeson S. 1996. Programas de alimentación para ponedoras y broilers. On line: http://www.etsia.upm.es/fedna/ capitulos/96capituloXI.pdf.

8. Leeson S, Summers JD. 1997. Commercial Poultry Nutrition, $2^{\text {nd }}$ ed., Ed. University Books, Guelph (Canada), p. 207-254.

9. Madrigal SE, Watkins NB, Wall A, Fritts CE, Waldroup PW. 2002. Effect of dietary modifications designed to reduce early growth rate on live performance and on incidence and severity of ascites in two commercial broiler strains when maintained under low ventilation or low temperature models. Poultry Sci 5: 150-157.

10. Meluzzi A, Cristofori C, Sirri F, Giordani G. 1995. Low sodium and low density diets to reduce broiler obesity. Zoot Int 6: 41-50.

11. Nocciolli HF, Kazue SN, Neme R, Rodrigues Freitas E. 2004. Níveis protéicos na ração de frangos de corte na fase de crescimento. Pesq Agropec Bras 39: 407-412. 
12. Penz AM. 1998. Avances en la alimentación de monogástricos: aves. On line: http://www.etsia.upm.es/fedna/ capitulos/98CAPXV.pdf.

13. Poole R. 1974. An introduction to quantitative ecology, McGraw-Hill, New York, p. 292-324.

14. Sakomura NK, Silva R. 1998. Conceitos aplicáveis à nutrição de não ruminantes. Cadernos Técnicos da Escola de Vetrinária da UFMG, Belo Horizonte, 22: 125-146.

15. Sandoval GL, Terraes JC, Fernández RJ, Revidatti FA, Campos MV, Quevedo HD. 1999. Perfil bioquímico sérico y peso corporal en pollos con estrés físico inducido y hepatoprotección continua. Memorias del XVI Congreso Latinoamericano de Avicultura, Lima (Perú), p. 4-7.

16. Santomá G. 1994 Programas de alimentación en broilers y "pollo alternativo". On line: http://www.etsia.upm.es/fedna/capitulos/94Cap_XIV.pdf.
17. Terraes JC, Sandoval GL, Fernandez RJ, Revidatti FA. 2001. Respuesta a una maniobra inductora de estrés y al tratamiento con un producto hepatoprotector en pollos de engorde. Vet Méx 32: 195-200.

18. Walker AW, Wiseman J, Lynn NJ, Chades DR. 1995. Recent findings on the effects of nutrition on the growth of specific broiler carcass components. In: Recents advances in animal nutrition (Garnsworthy PC, Cole DJ, Ed.), Nottingham University Press, Nottingham (UK), p. 169-184.

19. Zubair AK, Leeson S. 1994. Effect of varying period of early nutrient restriction on growth compensation and carcass characteristics of male broilers. Poultry Sci 73: 129136.

\section{Asociación Cooperadora de la Facultad de Ciencias Veterinarias}

Personería Jurídica No $647 / 92$ y $912 / 00$

Sargento Cabral 2139

3400 Corrientes

La Asociación Cooperadora de la Facultad de Ciencias Veterinarias fue constituida el 10 de diciembre del año 1991 como entidad de bien público, con el objeto de promover y coadyuvar las actividades científicas, educativas y culturales relacionadas con las Ciencias Veterinarias. En tal sentido, implementa acciones para colaborar con la enseñanza, extensión, actualización y difusión científica que realiza dicha Casa de Estudios.

Beneficios que brinda a sus asociados:

- Fotocopias con descuentos especiales del $20 \%$ en la Fotocopiadora COPIAS.COM que funciona dentro del predio de la Facultad de Ciencias Veterinarias.

- $10 \%$ de descuento para la adquisición de libros de la Editorial Inter-Médica.

- $10 \%$ de descuento en las compras de medicamentos e insumos para trabajos prácticos hospitalarios. 